\title{
ON THE THEORY OF THE DIRECTIONAL DISTRIBUTION AND THE POLARIZATION OF BETA AND GAMMA RAYS EMITTED BY ORIENTATED NUCLEI
}

\author{
by $H$. A. TOLHOEK
}

Instituut voor theoretische natuurkunde, Universiteit, Utrecht, Nederland

Several phenomena may be studied if nuclei are orientated in external or atomic fields at very low temperatures. We will discuss here the influence of such orientation on the $\beta$-and $\gamma$-rays emitted by radioactive nuclei. In the first place, the directional distribution of the emitted radiation may differ from spherical symmetry once the nuclei are orientated. Another phenomenon is that the radiation may show polarization phenomena. Both effects may occur for $\beta$-as well as for $\gamma$-radiation.

In certain cases we can readily show by simple arguments that the $\beta$-rays emitted by orientated nuclei may be polarized. Assuming non-relativistic energies for the emitted electron, the orbital and spin angular momentum of the electron can be considered separately. We know that for allowed transitions the electron is emitted without orbital angular momentum, while the neutrino takes the angular momentum $\frac{1}{2}$. If the initial nuclei are completely orientated $\left(m_{i}=j_{i} ; j_{i}\right.$ and $j_{f}$ characterize the nuclear spin of the initial and final nucleus respectively), it follows from the law of conservation of angular momentum that the $\beta$-rays must be totally polarized if $j_{i}=j_{i}+1$ (cf. Fig. 1). We have given a quantitative treatment of the polarization of $\beta$-rays in allowed transitions in an earlier paper ${ }^{1}$ ), and showed further that the directional distribution of the $\beta$-radiation remains spherically symmetric for allowed transitions ${ }^{1}$ ). However, for certain forbidden transitions the directional distribution may differ from spherical symmetry. (namely those forbidden transitions for which also $\beta-\gamma$ directional correlation may occur if a $\gamma$-ray exists $\left.{ }^{2}\right)$ ). 
(a) $j_{i}=j_{f}+1$

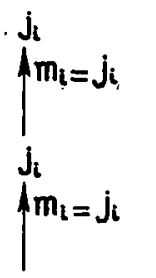

(b) $j_{i}=j_{f}$

(c) $\left.j_{i=j_{f}-1}\right|^{j_{i}} \quad \begin{aligned} & j_{f} m_{f}=j_{f} \\ & m_{i}=j_{i}\end{aligned} \quad \begin{array}{ll}\text { or } j_{f}-1 \\ \text { or } j_{f}-2\end{array}$

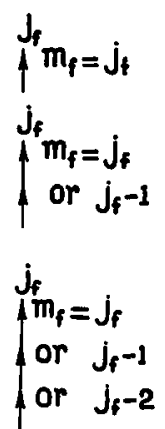

total $+4$ polarization. $\begin{array}{lll}i & t & 1 \\ e v & e & e\end{array}$ partial polarization.

$$
\text { ev ev ev ev polarization. }
$$

Fig. 1. The arrows indicate the possible values of the $z$-components of the angular momenta of the initial nucleus, the final nucleus, the electron $(e)$ and the neutrino $(v)$.

The $\gamma$-radiation will in general be emitted after a $\beta$-transition of the orientated initial nucleus. This $\beta$-transition will usually leave the nucleus appreciably orientated. In order to obtain the formulae for the directional distribution and polarization of the $\gamma$-radiation, we must study: a) the magnitude of these effects for a given orientation of the nucleus from which the $\gamma$-radiation is emitted and $b$ ) the change in orientation of a nucleus caused by a preceding $\beta$-(or $\gamma$-) transition. We have derived formulae; which give the solutions to these problems for arbitrary multipole order ${ }^{3}$ ), and for dipole and quadrupole transitions we have evaluated the general expressions so that they are ready for numerical calculations $\left.{ }^{4}\right)$. If $W(\vartheta)$ is the intensity of the radiation emitted at an angle $\vartheta$ to a direction of rotational symmetry $\eta$, the general formula for $2^{L}$-pole radiation has the form

$$
\frac{1}{2} W(\vartheta)=1+c_{2} t_{2} P_{2}(\cos \vartheta)+\ldots+c_{2 L} t_{2 L} P_{2 L}(\cos \vartheta) .
$$

This is deduced with the aid of R a c a h's algebra of tensoroperators ${ }^{5}$ ). Here $P_{k}(\cos \vartheta)$ is a Legendre polynomial, $c_{k}$ depends only on $k$ and the spin of the nucleus, and $f_{k}$ characterizes the orientation. We have, for example, (summation over $m$ from $-j$ to $j$; see also ${ }^{6}$ ))

$$
\begin{aligned}
& f_{1}=(1 / j) \Sigma_{m} m a_{m}, \\
& f_{2}=\left(1 / j^{2}\right)\left[\Sigma_{m} m^{2} a_{m}-\frac{1}{3} j(j+1)\right] .
\end{aligned}
$$

where $a_{m}$ gives the relative population of the different $m$-sublevels 
( $m$ with respect to $\eta, \Sigma_{m} a_{m}=1$ ). These parameters $f_{k}$ can also be used in the formulae for $\beta$-radiation. An important property of the $f_{k}$ 's is that $f_{k}=0$ if $k>2 j$; hence the highest $P_{2 k}(\cos \vartheta)$ involved in (1) is determined by the smallest of the numbers $2 j$ and $2 L$.

We now list the different phenomena, indicating whether the lowest $f_{k}$ that occurs in the formula for the effect is $f_{1}$ or $f_{2}$ :

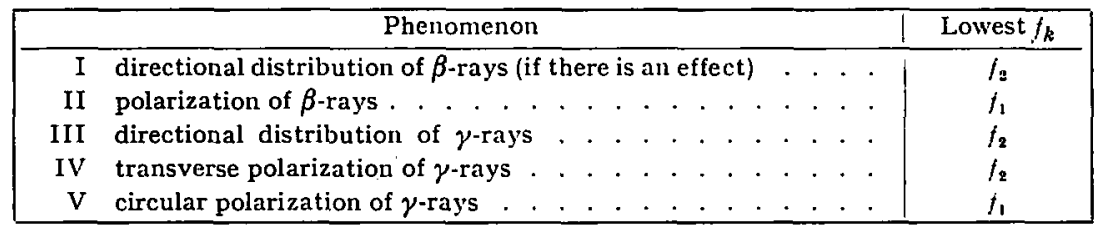

These results can be found without any calculation, just by considering the symmetry properties for the reflection of space in relation to the axial and polar vectors characterizing the different physical quantities. If we have nuclei with orientations represented by $+m$ and $-m$, the resulting quantities for the emitted radiation would be the same for I, III and IV, but for both orientations they would differ for II and V.

If the orientation of the nuclei is small, e.g. represented by a Boltzmann distribution in a magnetic field, $a_{m} \sim e^{\beta m}$ with small $\beta=\mu B / k T j$ ( $\mu$ magnetic moment, $B$ magnetic field), $f_{1} \ll f_{2}$. However, the orientation which has been attained in some experiments is quite appreciable.

As far as concerns the change of the orientation of the nuclei by a preceding $\beta$-transition, we can give formulae relating the $f_{k}$ before and after the $\beta$-transition. It is found that the connection depends on the order of forbiddenness of the $\beta$-transition, on the change of the nuclear spin, and in some cases also on the exact form for the Hamiltonian representing the $\beta$-interaction. For the general formulae the $\mathrm{R} \mathrm{a} \mathrm{c} \mathrm{a} \mathrm{h}$ algebra ${ }^{5}$ ) has again been used.

As to the experimental methods for observing the phenomena, we remark that observation of the $\beta$-rays is more difficult than observation of the $\gamma$-rays, since they are easily absorbed in the apparatus maintaining the low temperature. For the polarizatiou of the $\beta$-rays the additional difficulty exists that no "polarization detector of high efficiency" is known (detection of the polarization 
with scattering experiments gives a large loss by intensity). Transverse polarization of $\gamma$-radiation can be detected by measuring its Compton scattering in different directions. For circular polarization, Compton scattering on matter with polarized electrons (e.g. magnetized iron) is needed, which is more difficult to study.

We now survey the results which may be obtained in nuclear physics by measuring these effects:

1) If several possibilities exist for the values of the nuclear spins, the ambiguity may be decreased or eliminated.

2) Magnetic moments of radioactive nuclei can be determined. The ordinary methods are difficult to apply, since in most cases the radioactive nuclei are available only in small quantities. For the determination of the absolute magnitude, the directional distribution of $\gamma$-rays is sufficient; for the sign of the magnetic moment it is necessary to determine the sign of the circular polarization of $\gamma$-rays.

3) The multipole order of . $\gamma$-radiation can be deduced from the directional distribution. This is easiest for $\gamma$-rays of not too low energy. In order to decide whether one has electric or magnetic $2^{L}$-pole radiation, the sign of the transverse polarization must be measured.

4) As the change of the orientation by a preceding $\beta$-radiation depends on the Hamiltonian for the $\beta$-interaction, information on this Hamiltonian can be obtained by observation of a succeeding $\gamma$-radiation. Such information could also be obtained if the polarization of $\beta$-rays were observed.

These methods would gain in importance if the experimental methods for orientating nuclei could be extended to a larger number of nuclei than is at present possible.

These theoretical developments were made at the University of Utrecht in collaboration with Prof. S. R. de Groot and J. A. M. $\left.\left.\left(\mathrm{ox}^{1}\right)^{3}\right)^{4}\right)^{6}$ ) ${ }^{7}$ ). They were stimulated by the cooperation with the experimental work in Leiden by Prof. C. J. G orter, O. J. Poppema, M. J. Steenland, and J. A. B e u ${ }^{8}$ ): 


\section{REFERENCES}

1) Tol h o e k, H. A., Thesis, Utrecht, 1951, chapters II, III, IV, or Tol ho e k, H. A. and De Groot, S. R., Physica 17 (1951) 1, 17, 81.

2) Falkoff, D. L. and Uhle n beck, G. E., Phys. Rev. 79 (1950) 334.

3) Tol hoek, H. A. and Cox, J. A. M., Physica (to appear shortly).

4) Tol hoek, H. A. and Cox, J. A. M., Physica 18 (1952) 357.

5) R a c a h, G., Phys. Rev. B2 (1942) 438.

6) De Groot, S. R., Communication at this Conference, Physica 18 (1952) 1011.

7) $\mathrm{C} \circ \mathrm{x}$, J. A. M., Communication at this Conference, Physica 18 (1952) 1262.

8) P o p p e ma, O. J., Communication at this Conference, Physica 18 (1952) 1235. 